\title{
Evaluación de la densidad de plantas, componentes fenológicos de producción y rendimiento de granos en diferentes materiales genéticos de maíz
}

\author{
Evaluation of plant density, phenological components of production \\ and grain yield in different corn genotypes
}

Raúl Andrés Martínez Uribe ${ }^{1}$, Oriel Tiago Kölln², Glauber José de Castro Gava ${ }^{3}$

\section{RESUMEN}

El objetivo del estudio fue verificar la influencia de la distancia de plantación y de la población de plantas, en los componentes fenológicos y en la productividad de diferentes materiales genéticos de maíz. El experimento fue conducido en el municipio de Jaú/SP, localizado en la latitud $22^{\circ} 17^{\prime} \mathrm{S}$, longitud $48^{\circ} 34^{\prime} \mathrm{W}$ y altitud media de $680 \mathrm{msnm}$. Fueron estudiadas dos distancias de plantación: $0,45 \mathrm{~m}$ y $0,90 \mathrm{~m}$, tres densidades poblacionales 50, 75 y 100 mil plantas ha ${ }^{-1}$, y cuatro materiales genéticos: híbrido simple DKB 333C, híbrido triple DKB 466, híbrido doble AG 2060 y la variedad AL Bandeirantes ${ }^{\circledR}$ en esquema factorial $2 \times 3 \times 4$ con cuatro repeticiones en bloques al azar. La reducción en la distancia entre líneas de plantío y el aumento de la población de plantas influenciaron significativamente en el porcentaje de plantas acamadas y quebradas del maíz. Espacio entre líneas de $0,45 \mathrm{~m}$, población de 75.000 plantas $\mathrm{ha}^{-1}$ y material híbrido simple presentaron la mayor productividad de granos con promedio de $9.077 \mathrm{~kg} \mathrm{ha}^{-1}$. Se observaron interacciones significativas de los factores distancia entre líneas versus población de plantas, y población de plantas versus materiales para el factor productividad de granos. Los componentes fenológicos de producción: largo de la mazorca, diámetro de la mazorca y diámetro de la tusa (marlo) fueron influenciados por el aumento de población de plantas ha ${ }^{-1}$.

Palabras clave: Zea mays, población de plantas, híbridos.

\begin{abstract}
The aim of this study was to verify the influence of planting distance and the population of plants, phenological components, and productivity in different maize genetic material. The experiment was conducted during the 2003/04 agricultural year in the city of Jaú/SP, located at latitude $22^{\circ} 17^{\prime} \mathrm{S}$, longitude $48^{\circ} 34^{\prime} \mathrm{W}$ and altitude $680 \mathrm{~m}$. Average Were studied, planting distances of $0.45 \mathrm{~m}$ and $0.90 \mathrm{~m}$, three densities 50, 75 and 100000 plants ha ${ }^{-1}$, and four genetic materials: simple hybrid DKB 333C, triple hybrid $D K B$ 466, AG 2060 and double hybrid the variety AL 2x3x4 factorial scheme Bandeirantes in with 4 replications in randomized blocks. The reduction in the planting row spacing and increasing plant population significantly influenced the percentage of lodged plants and broken corn. Line spacing of $0.45 \mathrm{~m}$, population of 75,000 plants ha-1 and single hybrid material showed the highest productivity of grains with an average of $9077 \mathrm{~kg} \mathrm{ha}^{-1}$. Significant interactions were observed line spacing factors versus population of plants and plant population versus materials for grain productivity factor. Phonological components of production: spike length, shank diameter and the diameter of the cobs were influenced by increased plant population ha ${ }^{-1}$. The experiment was conducted during the agricultural year of 2003/04 in the city of Jaú/SP, located at latitude 22 $2^{\circ} 17^{\prime} S$ and longitude $48^{\circ} 34^{\prime} \mathrm{W}$ and an average altitude of $680 \mathrm{~m}$. Were tested, two spacing $0.45 \mathrm{~m}$ and $0.90 \mathrm{~m}$, three densities 50,75 and 100 thousand plants ha-1, and four genetic materials simple hybrid DKB 333C, triple hybrid DKB 466, double hybrid and variety AG 2060 in AL Bandeirantes ${ }^{\circledR}$ factorial design $2 \times 3 \times 4$ with 4 replications in randomized blocks.
\end{abstract}

Key words: Zea mays, plant population, hybrids.

\footnotetext{
Faculdade de Ciências e Engenharia - Tupã. UNESP -Universidade Estadual Paulista "Julio de Mesquita Filho", SP-Brasil.

2 CTBE, Campinas, SP-Brasil.

3 APTA, Pólo Regional do Centro Oeste, Jaú, SP-Brasil.

* Autor para correspondencia: raul@tupa.unesp.br
}

Fecha de Recepción: 16 Febrero, 2017.

Fecha de Aceptación: 30 Junio, 2017. 


\section{Introducción}

La cadena productiva del maíz (Zea mays L.) en Brasil representa elevada importancia socioeconómica en función de su potencial productivo y valor nutritivo. El es uno de los principales insumos para el segmento del agronegocio, siendo ampliamente utilizado en la avicultura (31\%); porcinocultura (14\%); bovinocultura $(4,5 \%)$; para el sector industrial (7,68\%); y para el consumo humano (1,9\%) en la alimentación (Abimilho, 2016).

Según la Companhia Nacional do Abastecimento (CONAB) en la cosecha 2014/2015 el área plantada fue 15,74 millones de ha, con una producción de 84,7 millones de toneladas, y una productividad media de 5381,8 $\mathrm{kg} \mathrm{ha}^{-1}$. Existe una elevada discrepancia entre el potencial productivo del maíz de 10 t ha-1 y el observado en la práctica de 2 a 4 t ha $^{-1}$ de granos (Coelho \& França, 1995).

Varias causas contribuyen para la obtención de estos bajos rendimientos, destacándose principalmente el uso de variedades con bajo potencial productivo, deficiencia hídrica, baja fertilidad de los suelos cultivados, implantación inadecuada de época y densidad de siembra y control inadecuado de insectos y plantas dañinas (Silva et al., 2003; Fancelli y Dourado Neto, 2000).

Llevando en cuenta que Brasil dispone de semillas con buena calidad fitosanitaria y buenos atributos genéticos, así como óptimas condiciones de radiación para el proceso fotosintético, se considera que la baja productividad se debe al mal manejo del cultivo para las condiciones edafoclimáticas reinantes (Fonasieri Filho, 1992).

El interés en cultivar maíz utilizando espaciamientos entre líneas reducidos, 45 a $60 \mathrm{~cm}$, ha crecido en los últimos años en diferentes regiones productoras, principalmente entre los productores que trabajan con densidades de siembra superiores a 50.000 plantas ha ${ }^{-1} \mathrm{y}$ alcanzan rendimientos de granos superiores a $6.000 \mathrm{~kg} \mathrm{ha}^{-1}$ (Sangoi et al., 2001). Sin embargo, los efectos de la reducción del espaciamiento entre líneas sobre el rendimiento de granos del maíz existentes en la literatura son bastante heterogéneos. En el sur del Brasil, incrementos de 5 a $8 \%$ fueron reportados por Sangoi et al. (2001) y Peixoto (2002) con la reducción en el espaciamiento de $90-100 \mathrm{~cm}$ a $45-50 \mathrm{~cm}$. En el Cerrado Brasilero, los beneficios reportados por la reducción del espaciamiento entre líneas de 90 a $45 \mathrm{~cm}$ son porcentualmente mayores que en el sur del país, oscilando entre 9 y $41 \%$, dependiendo de la densidad, cultivar y año agrícola (Fundación Rio Verde, 2002).

En virtud de las modificaciones introducidas en los genotipos de maíz más recientes, como menor estatura de la planta y altura de inserción de la mazorca, menor esterilidad de plantas, menor duración del subperíodo florecimientoespigamiento, plantas con hojas de ángulo más recto y elevado potencial productivo, se torna necesario reevaluar las recomendaciones de espaciamiento y densidad de siembra para el cultivo del maíz (Argenta et al., 2001).

Por tanto, el objetivo del trabajo fue verificar la influencia del espaciamiento y la población de plantas, en los componentes fenológicos y en la productividad de diferentes materiales genéticos de maíz.

\section{Materiales y Métodos}

El experimento fue instalado en el municipio de Jaú-SP/Brasil, localizado en la latitud $22^{\circ} 17^{\prime} \mathrm{S}$, y longitud $48^{\circ} 34^{\prime}$ 'W y altitud media de $680 \mathrm{msnm}$. El clima predominante en la región según Köppen es Aw con clima seco definido, temperatura media anual de $21,6{ }^{\circ} \mathrm{C}$, humedad relativa media de $70 \%$, con extremos de $77 \%$ en febrero y $59 \%$ en agosto, y un promedio pluviométrico de $1.344 \mathrm{~mm}$. El suelo fue clasificado como Nitossolo Vermelho distoférrico (Embrapa, 1999). Los atributos químicos del suelo, según metodología descrita por Raij (1996), en la profundidad de 0-20 cm fueron: $\mathrm{P}$ (resina): $31 \mathrm{mg} \mathrm{dm}^{-3}$; M.O.: $32,6 \mathrm{~g} \mathrm{dm}^{-3} ; \mathrm{pH}\left(\mathrm{CaCl}_{2}\right): 5,3 ; \mathrm{K}: 1,6 \mathrm{mmol}_{\mathrm{c}} \mathrm{dm}^{-3}$; Ca: 25,3 mmol $_{\mathrm{c}} \mathrm{dm}^{-3} ; \mathrm{Mg}: 9 \mathrm{mmol}_{\mathrm{c}} \mathrm{dm}^{-3} ; \mathrm{H}+\mathrm{Al}$ : $30,1 \mathrm{mmol}_{\mathrm{c}} \mathrm{dm}^{-3}$; SB: $35,9 \mathrm{mmol}_{\mathrm{c}} \mathrm{dm}^{-3}$, CTC: $66,0 \mathrm{mmol}_{\mathrm{c}} \mathrm{dm}^{-3}$ y V: $59,4 \%$.

El diseño experimental fue en bloques al azar, con parcelas sub-subdivididas con 24 tratamientos dispuestos en esquema factorial $2 \times 3 \times 4$, con cuatro repeticiones. Las parcelas principales fueron constituidas de dos espacios entre líneas $(0,90$ y $0,45 \mathrm{~m}$ ); divididas en tres densidades (50, $75 \mathrm{y}$ 100 mil plantas ha ${ }^{-1}$ ); formando subparcelas con cuatro materiales (híbrido simple, híbrido triple, híbrido doble y una variedad). Se utilizó el híbrido simple (HS) DKB® 333C, híbrido triple (HT) DKB® 466, híbrido doble (HD) AG 2060 y la variedad (V) AL Bandeirantes ${ }^{\circledR}$. Las subparcelas fueron constituidas de ocho líneas con cinco metros 
de largo. Como área útil fueron consideradas las seis líneas centrales, desconsiderando un metro en cada extremo.

El experimento fue conducido en el sistema convencional, considerando que el cultivo anterior al plantío fue avena, se realizó la fertilización en el surco de siembra utilizándose la fórmula 08-28-16, en la dosis $300 \mathrm{~kg} \mathrm{ha}^{-1}$. La siembra fue realizada manualmente, siguiendo la distribución de acuerdo con la población deseada, utilizando un exceso de $50 \%$ de semillas. La brotación ocurrió siete días después de la siembra (DDS) y el desbaste (raleo) fue realizado 13 días después de la brotación (DDB), dejándose el número de plantas por metro correspondiente a la población del respectivo tratamiento. Para los casos donde ocurrieron densidades de plantas diferentes de las propuestas fue realizada la corrección por el método de la covariancia (Ramalho, 2000).

El control de plantas invasoras fue realizado por la aplicación de herbicida postemergente con el principio activo nicosulfuron en la dosis 1,5 $1 \mathrm{ha}^{-1}$ del producto comercial. Para el control del gusano cogollero (Spodopthera frugiperda), se utilizó producto a base de trencer con dosis de $50 \mathrm{ml} \mathrm{ha}^{-1}$, aplicado directamente en el interior del cogollo con pulverizador costal equipado con una boquilla tipo abanico 80-04.

A los 39 DDB, con siete hojas totalmente desdobladas, se realizó la fertilización nitrogenada en cobertura, en la dosis de $250 \mathrm{~kg} \mathrm{~N}^{-1}$, usando como fuente de nitrógeno la urea.
Fueron evaluados los siguientes componentes: acamado y quebrado: contadas, en el área útil de la sub-subparcela, como acamadas plantas que presentaban ángulo de inclinación superior a $45^{\circ}$ con la vertical y como quebradas plantas quebradas debajo de la espiga superior, en porcentaje. Diámetro de la tusa (marlo) y de la mazorca en una muestra de doce colectadas por sub-subparcela: determinado por medio de vernier en centímetros. Largo de la mazorca: determinado por la distancia entre la base hasta la punta en centímetros. La productividad de granos: obtenida por el peso de granos del área útil de cada subparcela, determinándose la proporción de agua por el método gravimétrico en $\mathrm{kg} \mathrm{ha}^{-1}$ (13\% de humedad).

Para interpretar mejor los resultados, con base en datos obtenidos de la estación meteorológica localizada en Jaú-APTA, se realizó el balance hídrico secuencial decendial según PenmanMonteith (Figura 1), además de la temperatura media y radiación global decendiales (Figura 2). Para el análisis estadístico de las evaluaciones se utilizó el experimento factorial con tres factores (espaciamientos, población de plantas y materiales) con delineamiento en bloques al azar, los promedios fueron comparados por el test de Tukey $(\mathrm{p}<0,05)$.

\section{Resultados y Discusión}

Conforme puede observarse en los resultados presentados en la Tabla 1, el factor espaciamiento entre línea no influyó significativamente en el número

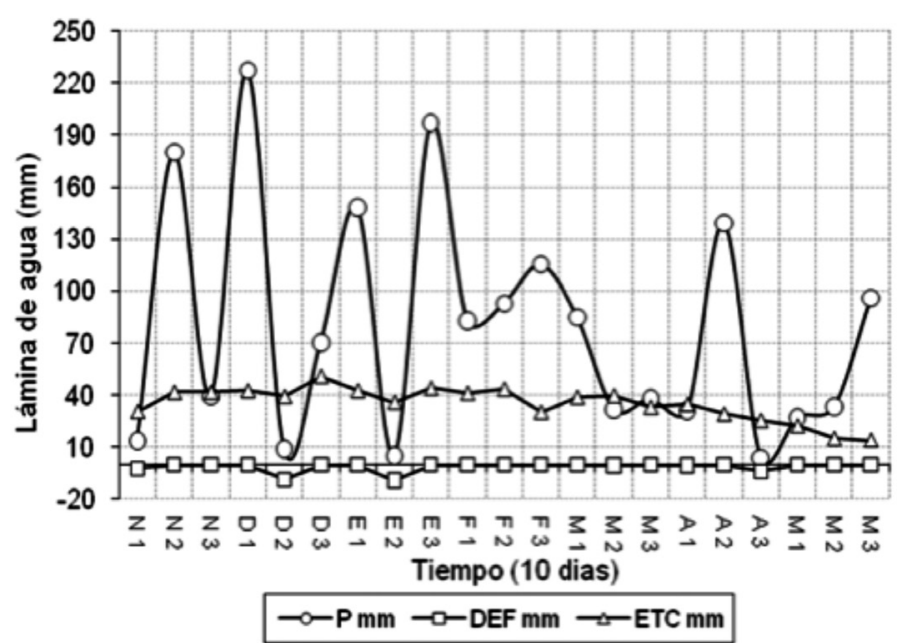

Figura 1. Balance hídrico secuencial referente al período de noviembre de 2013 a mayo de 2014. 


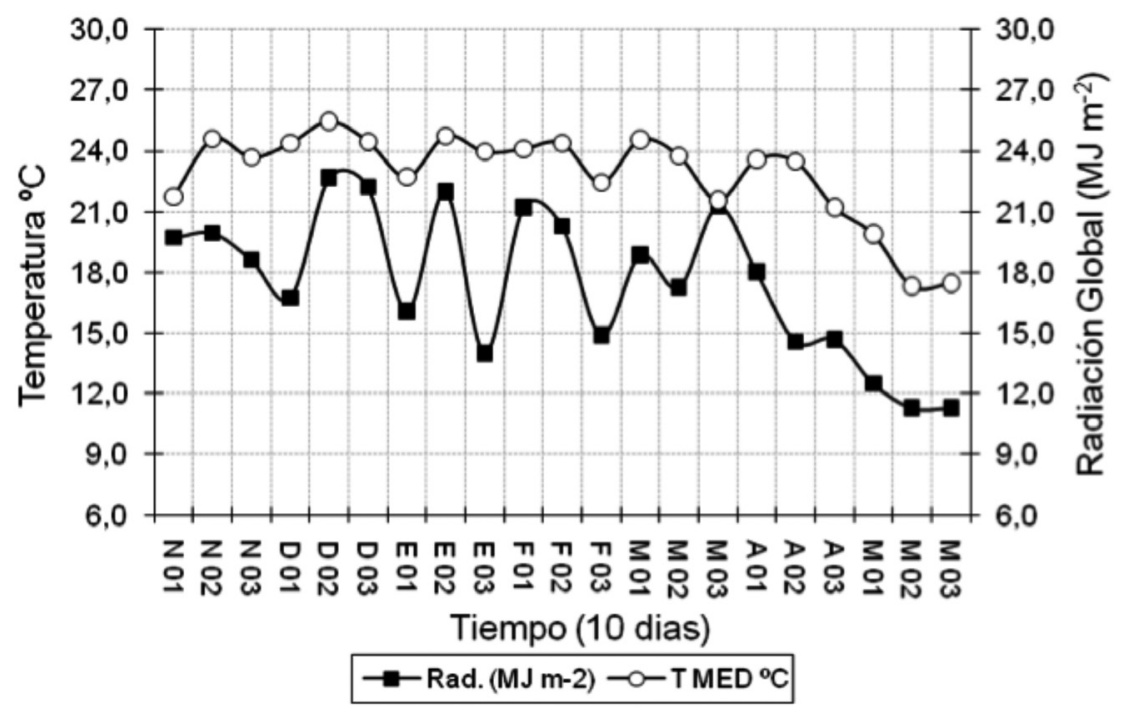

Figura 2. Temperatura media y radiación global en el período de noviembre de 2013 a mayo de 2014.

Tabla 1. Valores medios relativos al porcentaje de plantas quebradas y acamadas, en materiales de maíz sometido a diferentes arreglos de stand.

\begin{tabular}{lcc}
\hline \multicolumn{1}{c}{ Causas de la variación } & $\begin{array}{c}\text { Plantas } \\
\text { quebradas }\end{array}$ & $\begin{array}{c}\text { Plantas } \\
\text { acamadas }\end{array}$ \\
\hline Espaciamiento E $(\mathrm{m})$ & $\%$ & $\%$ \\
\hline $0,90\left(\mathrm{E}_{1}\right)$ & $2,42 \mathrm{~b}$ & $2,13 \mathrm{a}$ \\
$0,45\left(\mathrm{E}_{2}\right)$ & $5,92 \mathrm{a}$ & $1,56 \mathrm{a}$ \\
Test F & $36,20^{*}$ & $1,25^{\mathrm{ns}}$ \\
**Dms (Tukey) a 5\% & 401,16 & 1,01 \\
\hline Población de plantas P (plantas ha-1) & & \\
\hline 100.000 plantas/ha & $7,56 \mathrm{a}$ & $3,56 \mathrm{a}$ \\
75.000 plantas/ha & $3,88 \mathrm{~b}$ & $1,72 \mathrm{~b}$ \\
50.000 plantas/ha & $1,06 \mathrm{c}$ & $0,25 \mathrm{~b}$ \\
Test F & $41,87^{*}$ & $14,49^{*}$ \\
Dms (Tukey) a 5\% & 1,71 & 1,48 \\
\hline Materiales & & \\
\hline HS & $1,08 \mathrm{c}$ & $0,46 \mathrm{c}$ \\
HT & $3,79 \mathrm{~b}$ & $1,26 \mathrm{bc}$ \\
HD & $7,21 \mathrm{a}$ & $2,42 \mathrm{ab}$ \\
V & $4,58 \mathrm{~b}$ & $3,25 \mathrm{a}$ \\
Test F & $18,79^{*}$ & $6,02^{*}$ \\
Dms (Tukey) a 5\% & 2,17 & 1,88 \\
\hline Interacción E X P & $9,8^{*}$ & $0,48^{\mathrm{ns}}$ \\
Interacción E X M & $2,77^{*}$ & $1,08^{\mathrm{ns}}$ \\
Interacción P X M & $3,45^{*}$ & $2,36^{*}$ \\
Interacción E X P X M & $2,09^{\mathrm{ns}}$ & $0,64^{\mathrm{ns}}$ \\
CV(\%) & 68,4 & 133,77 \\
\hline
\end{tabular}

ns: No significativo; *significativo a 5\% de probabilidad. Medias seguidas de la misma letra no difieren entre sí por el teste de Tukey $(\mathrm{P}>0,05)$, ** DMS: Diferencia minina significativa. HS: híbrido simple, HT: híbrido triple, HD: híbrido doble, V: variedad. de plantas acamadas, no obstante se nota que con el incremento en la población de plantas $\mathrm{ha}^{-1}$ ocurre un aumento del porcentaje de plantas acamadas en el cultivo del maíz, siendo que, el tratamiento de 75.000 y 50.000 plantas ha $^{-1}$ no difiere entre sí, pero difieren significativamente con el tratamiento de 100.000 plantas ha ${ }^{-1}$. El híbrido simple presenta menor número de plantas acamadas; sin embargo, no difieren estadísticamente del híbrido triple, mas difiere del híbrido doble y de la variedad.

Según Gross et al. (2006), el aumento de la densidad poblacional interfiere de forma significativa en la reducción de la masa individual de las plantas resultado de la competencia entre ellas por los recursos del medio. Con esto se puede inferir que las plantas tienden a ser más susceptibles al quebrado y/o acamado con el incremento poblacional, entre tanto ocurren diferencias entre genotipos (Demétrio et al., 2008).

La interacción entre población de plantas y materiales fue significativa (Tabla 2 ), se verifica que la población de 100.000 plantas ha $^{-1}$ presenta mayor número y el tratamiento de 50.000 plantas ha ${ }^{-1}$ menor número de plantas acamadas, siendo que el material híbrido simple presentó mayor resistencia al acamado de plantas que los demás materiales en todas las poblaciones evaluadas, las otras interacciones no presentaron significancia entre sí.

Estudiando el número de plantas quebradas, todos los factores presentaron influencias significativas (Tabla 1). Se observa que con la disminución del 
Tabla 2. Desdoblamiento de la interacción población de plantas versus materiales en el porcentaje de plantas acamadas del maíz.

\begin{tabular}{|c|c|c|c|c|}
\hline \multirow{3}{*}{ Población } & \multicolumn{4}{|c|}{ Materiales } \\
\hline & HS & HT & HD & $\mathrm{V}$ \\
\hline & \multicolumn{4}{|c|}{$-\%-$} \\
\hline $100.000 \mathrm{pl} \mathrm{ha}^{-1}$ & $1,00 \mathrm{Ca}^{*}$ & $1,75 \mathrm{BCa}$ & 4,75 $\mathrm{ABa}$ & $6,75 \mathrm{Aa}$ \\
\hline $75.000 \mathrm{pl} \mathrm{ha}^{-1}$ & $0,38 \mathrm{Aa}$ & $1,75 \mathrm{Aa}$ & $2,00 \mathrm{Aab}$ & $2,75 \mathrm{Ab}$ \\
\hline $50.000 \mathrm{pl} \mathrm{ha}^{-1}$ & $0,00 \mathrm{Aa}$ & $0,25 \mathrm{Aa}$ & $0,50 \mathrm{Ab}$ & $0,25 \mathrm{Ab}$ \\
\hline
\end{tabular}

*Las medias seguidas de la misma letra no difieren entre sí en las líneas (mayúsculas) y en la columna (minúsculas) por el test de Tukey a 5\% de probabilidad. HS: híbrido simple, HT: híbrido triple, HD: híbrido doble, V: variedad.

espaciamiento y el aumento de la población de plantas ocurre un incremento en plantas quebradas. En relación a los materiales el híbrido doble presenta mayor caída, siendo significativa en relación a los otros materiales, el híbrido triple y la variedad no difieren entre sí y difieren de los demás materiales. Se puede apreciar el efecto de las interacciones entre población de plantas versus materiales, espaciamiento entre líneas versus materiales y espaciamiento entre líneas versus población de plantas (Tabla 3).

En la interacción entre población y materiales se verifica que a medida que ocurre el aumento de la población en forma simultánea aumenta el número de plantas quebradas en todos los materiales, siendo el material híbrido simple el de mayor resistencia al quiebre de plantas. En investigaciones realizadas por Barbosa (1995), los espaciamientos ejercieron influencia significativa sobre las características de acamado y quiebra de plantas de maíz y los estudios de regresión revelaron efecto linear sobre las plantas acamadas. Se observó también que, con el aumento de los espaciamientos ocurrió una disminución progresiva y linear en el número de plantas acamadas. En relación a la interacción espaciamiento y población, cuando ocurre aumento en el número de plantas ha-1 y reducción en el espaciamiento, se eleva el número de plantas quebradas. Evaluándose la interacción espaciamiento y materiales se observa que el material híbrido simple presenta menor número de plantas quebradas, al contrario del híbrido doble que presenta los mayores valores de plantas quebradas en los dos espaciamientos estudiados.

En la productividad de granos, ocurrieron diferencias significativas para el factor espaciamiento entre líneas, destacándose el mejor resultado en el espaciamiento de $0,45 \mathrm{~m}\left(7.161,67 \mathrm{~kg} \mathrm{ha}^{-1}\right)$. Según Argenta (2001), el cultivo del maíz responde linealmente a la reducción del espaciamiento, estando relacionado aún con la densidad poblacional aplicada. En lo que se refiere a población de plantas ha ${ }^{-1}$ no hubo significancia entre las 100.000 y 75.000 plantas $a^{-1}$; ocurriendo una diferencia

Tabla 3. Desdoblamiento de las interacciones entre población de plantas versus materiales, espaciamiento versus materiales, y espaciamiento versus población en el porcentaje de plantas quebradas del maíz.

\begin{tabular}{|c|c|c|c|c|}
\hline \multirow{3}{*}{ Población } & \multicolumn{4}{|c|}{ Materiales } \\
\hline & HS & HT & HD & V \\
\hline & \multicolumn{4}{|c|}{$-\%-$} \\
\hline $100.000 \mathrm{pl} \mathrm{ha}^{-1}$ & $1,88 \mathrm{Ba}^{*}$ & $8,63 \mathrm{Aa}$ & $11,50 \mathrm{Aa}$ & $8,25 \mathrm{Aa}$ \\
\hline $75.000 \mathrm{pl} \mathrm{ha}^{-1}$ & $1,13 \mathrm{Ba}$ & $2,25 \mathrm{Bb}$ & $7,88 \mathrm{Ab}$ & $4,25 \mathrm{ABb}$ \\
\hline $50.000 \mathrm{pl} \mathrm{ha}^{-1}$ & $0,25 \mathrm{Aa}$ & $0,50 \mathrm{Ab}$ & $2,25 \mathrm{Ac}$ & $1,25 \mathrm{Ab}$ \\
\hline EspaciamientoE (m) & HS & HT & $\mathrm{HD}$ & V \\
\hline 0,90 & $0,17 \mathrm{Ca}$ & $1,33 \mathrm{BC} \mathrm{b}$ & $4,50 \mathrm{Ab}$ & 3,67 ABa \\
\hline 0,45 & 2,00 Ca & $6,25 \mathrm{Ba}$ & 9,92 Aa & $5,50 \mathrm{Ba}$ \\
\hline \multirow[t]{2}{*}{ Espaciamiento E (m) } & \multicolumn{4}{|c|}{ Población } \\
\hline & $100.000 \mathrm{pl} \mathrm{ha}^{-1}$ & $75.000 \mathrm{pl} \mathrm{ha}^{-1}$ & \multicolumn{2}{|c|}{$50.000 \mathrm{pl} \mathrm{ha}^{-1}$} \\
\hline $0,90 \mathrm{~m}$ & $4,06 \mathrm{Ab}$ & $2,56 \mathrm{ABb}$ & \multicolumn{2}{|c|}{$0,63 \mathrm{Ba}$} \\
\hline $0,45 \mathrm{~m}$ & $11,06 \mathrm{Aa}$ & $5,19 \mathrm{Ba}$ & \multicolumn{2}{|c|}{$1,5 \mathrm{Ca}$} \\
\hline
\end{tabular}

*Las medias seguidas de la misma letra no difieren entre sí en las líneas (mayúsculas) y en la columna (minúsculas) por el test de Tukey a 5\% de probabilidad. HS: híbrido simple, HT: híbrido triple, HD: híbrido doble, V: variedad. 
significativa al compararse con el tratamiento de 50.000 plantas ha $^{-1}$ (Tabla 4).

Al comparar los materiales entre sí, se visualizó una mayor productividad del híbrido simple en relación a los demás híbridos y a la variedad. Se observaron también interacciones significativas entre los factores espaciamiento entre líneas versus población de plantas, y población de plantas versus materiales (Tabla 5).

En la interacción población de plantas versus materiales hay diferencia significativa en la productividad de granos entre los materiales híbrido simple y triple en las poblaciones de 100.000 y 75.000 plantas ha $^{-1}$, y ambas difirieron significativamente de la población de 50.000 plantas ha ${ }^{-1}$, corroborando con los resultados obtenidos por Amaral Filho et al. (2005).

El espaciamiento entre líneas de 0,45 m, población de 75.000 plantas ha $^{-1}$ y material híbrido simple presentaron la mayor productividad de granos con una media de $9.077 \mathrm{~kg} \mathrm{ha}^{-1}$. En función de las características genéticas de un híbrido simple, acerca de condiciones favorables de clima y suelo, la población indicada varía entre 60.000 a 80.000 plantas ha-1, pues de acuerdo con Peixoto et al. (1997), cuanto más favorables son las condiciones edafoclimáticas, mayor será la población necesaria para maximizar la producción de granos.

Las productividades obtenidas son semejantes con otras obtenidas en el estado de São Paulo, (Argenta et al., 2001; Amaral Filho et al., 2005; Borghi et al., 2006) y pueden ser explicadas por la fertilidad moderada del área experimental que tiene un buen nivel de materia orgánica y también por la distribución uniforme de las lluvias en ese período, que hicieron con que ocurriese una baja deficiencia hídrica de apenas $24 \mathrm{~mm}$ a lo largo del ciclo.

Tabla 4. Productividad, longitud de las mazorcas, diámetro de las mazorcas y diámetro de la tusa (marlo) en materiales de maíz sometidos a diferentes arreglos poblacionales.

\begin{tabular}{lcccc}
\hline \multirow{2}{*}{ Causas de la variación } & Productividad & Longitud mazorcas & Diámetro mazorca & Diámetro tusa \\
\cline { 2 - 5 } & \multicolumn{1}{c}{$\mathrm{kg} \mathrm{ha}^{-1}-$} & & $-\mathrm{cm}$ & \\
\hline Espaciamiento E $(\mathrm{m})$ & & & & \\
\hline $0,90\left(\mathrm{E}_{1}\right)$ & $6.786,75 \mathrm{~b}$ & $14,08 \mathrm{a}$ & $4,858 \mathrm{a}$ & $2,74 \mathrm{a}$ \\
$0,45\left(\mathrm{E}_{2}\right)$ & $7.161,67 \mathrm{a}$ & $14,11 \mathrm{a}$ & $4,85 \mathrm{a}$ & $2,72 \mathrm{a}$ \\
Test f & $11,36^{*}$ & $0,28^{\mathrm{ns}}$ & $0,03^{\mathrm{ns}}$ & $0,98^{\mathrm{ns}}$ \\
**Dms (tukey) a 5\% & 222,00 & 0,43 & 0,093 & 0,05 \\
\hline Población de plantas P (plantas ha-1) & & & \\
\hline 100.000 plantas/ha & $7.070,56 \mathrm{a}$ & $12,6 \mathrm{c}$ & $4,73 \mathrm{~b}$ & $2,65 \mathrm{c}$ \\
75.000 plantas/ha & $7.394,00 \mathrm{a}$ & $14,19 \mathrm{~b}$ & $4,87 \mathrm{a}$ & $2,72 \mathrm{~b}$ \\
50.000 plantas/ha & $6.458,06 \mathrm{~b}$ & $15,55 \mathrm{a}$ & $4,97 \mathrm{a}$ & $2,82 \mathrm{a}$ \\
Test f & $24,35^{*}$ & $61,40^{*}$ & $8,97^{*}$ & $19,29^{*}$ \\
**Dms (tukey) a 5\% & 326,55 & 0,63 & 0,14 & 0,07 \\
\hline Materiales & & & & \\
\hline HS & $7.987,82 \mathrm{a}$ & $14,85 \mathrm{a}$ & $4,71 \mathrm{~b}$ & $2,59 \mathrm{~b}$ \\
HT & $7.436,88 \mathrm{~b}$ & $13,24 \mathrm{~b}$ & $5,04 \mathrm{a}$ & $2,77 \mathrm{a}$ \\
HD & $6.462,08 \mathrm{c}$ & $14,22 \mathrm{a}$ & $4,87 \mathrm{ab}$ & $2,80 \mathrm{a}$ \\
V & $6.009,96 \mathrm{~d}$ & $14,08 \mathrm{a}$ & $4,8 \mathrm{~b}$ & $2,76 \mathrm{a}$ \\
Test f & $65,55^{*}$ & $9,4^{*}$ & $8,77^{*}$ & $15,88^{*}$ \\
Dms (tukey) a 5\% & 414,60 & 0,80 & 0,17 & 0,09 \\
\hline Interacción E x P & $3,49^{*}$ & $5,20^{\mathrm{ns}}$ & $3,1^{\mathrm{ns}}$ & $1,68^{\mathrm{ns}}$ \\
Interacción E x M & $0,57^{\mathrm{ns}}$ & $1,30^{\mathrm{ns}}$ & $0,43^{\mathrm{ns}}$ & $0,16^{\mathrm{ns}}$ \\
Interacción P x M & $5,00^{*}$ & $2,68^{\mathrm{ns}}$ & $0,76^{\mathrm{ns}}$ & $1,14^{\mathrm{ns}}$ \\
Interacción E x P x M & $0,68^{\mathrm{ns}}$ & $4,70^{\mathrm{ns}}$ & $1,17^{\mathrm{ns}}$ & $1,17^{\mathrm{ns}}$ \\
CV(\%) & 7,81 & 7,48 & 4,72 & 4,16 \\
\hline
\end{tabular}

ns: No significativo; *significativo a $5 \%$ de probabilidad medias seguidas de misma letra no difieren entre sí por el test de Tukey $(\mathrm{P}>0,05)$ ** DMS: Diferencia minina significativa. HS: híbrido simple, HT: híbrido triple, HD: híbrido doble, V: variedad. 
Tabla 5. Desdoblamiento de las interacciones: Espaciamiento entre líneas versus población de plantas, y población de plantas versus diferentes materiales en la productividad del maíz.

\begin{tabular}{|c|c|c|c|c|}
\hline \multirow{3}{*}{ Espaciamiento E (m) } & \multicolumn{4}{|c|}{ Población } \\
\hline & \multicolumn{4}{|c|}{$-\mathrm{Kg} \mathrm{ha}^{-1}-$} \\
\hline & $100000 \mathrm{pl} \mathrm{ha}^{-1}$ & \multicolumn{2}{|c|}{$75000 \mathrm{pl} \mathrm{ha}^{-1}$} & $50000 \mathrm{pl} \mathrm{ha}^{-1}$ \\
\hline $0,90 \mathrm{~m}$ & $6821,50 \mathrm{AB}^{*} *$ & \multicolumn{2}{|c|}{$7065,56 \mathrm{Ab}$} & $73,19 \mathrm{Ba}$ \\
\hline $0,45 \mathrm{~m}$ & $7319,63 \mathrm{Aa}$ & \multicolumn{2}{|c|}{$7722,44 \mathrm{Aa}$} & $42,94 \mathrm{Ba}$ \\
\hline \multirow{2}{*}{ Población de plantas $\mathrm{P}$ (plantas ha ${ }^{-1}$ ) } & \multicolumn{4}{|c|}{ Materiales } \\
\hline & HS & HT & HD & $\mathrm{V}$ \\
\hline $100000 \mathrm{pl} \mathrm{ha}^{-1}$ & $8489,86 \mathrm{Aa}$ & $7819,25 \mathrm{Aa}$ & $6258,38 \mathrm{Ba}$ & $5714,75 \mathrm{Bb}$ \\
\hline $75000 \mathrm{pl} \mathrm{ha}^{-1}$ & $8536,13 \mathrm{Aa}$ & $7773,13 \mathrm{Ba}$ & $6841,38 \mathrm{Ca}$ & $6425,38 \mathrm{Ca}$ \\
\hline $50000 \mathrm{pl} \mathrm{ha}^{-1}$ & $6937,75 \mathrm{Ab}$ & $6718,25 \mathrm{Ab}$ & $6286,50 \mathrm{ABa}$ & $5889,75 \mathrm{Bab}$ \\
\hline
\end{tabular}

*Las medias seguidas de la misma letra no difieren entre sí en las líneas (mayúsculas) y en la columna (minúsculas) por el test de Tukey a 5\% de probabilidad. HS: híbrido simple, HT: híbrido triple, HD: híbrido doble, V: variedad.

La productividad de granos fue menor con la utilización de espaciamiento entre líneas de 0,45 m, población de 100.000 plantas ha ${ }^{-1}$ y la variedad como material, con una media de $5.710 \mathrm{~kg} \mathrm{ha}^{-1}$; sin embargo, se mostró superior al último promedio (5.381,8 $\mathrm{kg} \mathrm{ha}^{-1}$ ) de la cosecha 2014/15 del estado de São Paulo (Conab, 2015).

Conforme los resultados presentados en la Tabla 5, el factor espaciamiento no influenció en el largo de las mazorcas, aunque a medida que aumenta la población de plantas ha- ${ }^{-1}$, ocurre una reducción significativa en el largo de las mismas. En relación a los materiales solamente el híbrido triple difiere significativamente entre los materiales presentando el menor valor. Dourado Neto et al. (2003) verificaron que en las poblaciones comprendidas entre 30 mil y 60 mil plantas por hectárea, los genotipos de maíz presentaron incremento del largo de mazorca. Según Sangoi et al. (2000), plantas espaciadas equidistantemente compiten mínimamente por nutrientes, luz y otros factores, favoreciendo el mejor desarrollo de las mazorcas.

Se observó con relación al diámetro de las mazorcas que los valores no presentaron diferencias significativas en relación al factor espaciamiento; sin embargo, el factor población de plantas arrojó diferencias significativas en la población de 100.000 plantas ha ${ }^{-1}$ en relación a las otras poblaciones. El híbrido triple presenta el mayor diámetro medio de mazorca, siendo significativo en relación al híbrido simple y la variedad, y el híbrido doble no presenta significancia en relación a los materiales.
El espaciamiento no influenció significativamente el diámetro de la tusa (marlo). En relación a la población de plantas ocurrió diferencia significativa, siendo que cuanto más grande es la población de plantas ha ${ }^{-1}$, menor el diámetro de la tusa (marlo). Con relación a los materiales, el híbrido simple presentó un valor medio menor y difiere significativamente de los demás materiales, que por su parte no presentaron significancia entre sí.

\section{Conclusiones}

Menor espaciamiento entre líneas generó mayor porcentaje de plantas quebradas; sin embargo, ese efecto no fue verificado en el factor plantas acamadas.

Fueron observados aumentos significativos en el porcentaje de plantas quebradas con el aumento de la población de plantas ha ${ }^{-1}$ en los materiales híbrido doble, híbrido triple y variedad.

La población de 50.000 plantas ha ${ }^{-1}$ presentó menores productividades en relación a las mayores poblaciones para todos los materiales.

Para los híbridos simple y triple hubo aumentos significativos de la productividad cuando la población fue de 75.000 y 100.000 plantas ha $^{-1}$, no fue verificado el mismo efecto con el híbrido doble y la variedad.

El material híbrido simple se mostró superior en las diferentes poblaciones y espaciamientos testados en relación a los demás materiales.

Los componentes longitud de mazorca, diámetro medio de mazorca y diámetro medio de la tusa (marlo) se mostraron sensibles al aumento de la población de plantas por hectárea. 


\section{Literatura Citada}

Abimilho

Associação Brasileira das indústrias do milho. http://www. abimilho.com.br/estatisticas Consultado 28 mar. 2017.

Amaral Filho, J.P.R. do; Fornasieri Filho, D.; Farinelli, R. e

Barbosa, J.C.

2005. Espaçamento, densidade populacional e adubação nitrogenada na cultura do milho. Revista Brasileira de Ciência do Solo, 29: 467-473.

Argenta, G.S.; Silva, P.R.F. da; Bortolini, C.G.; Forsthofer,

E.L.; Manjabosco, E.A. e Beheregaray Neto, V.

2001. Resposta de híbridos simples à redução do espaçamento entre linhas. Pesquisa Agropecuária Brasileira, 16 (1): 71-78.

Esechie, H.A.

1992. Effect of planting density on growth and yield of irrigated maize (Zea mays) in the Batinah Coast region of Oman. Journal of Agricultural Science, 119: 165-169.

Borghi, E.; Crusciol, C.A.C.; Costa, C.

2006. Desenvolvimento da cultura do milho em consorciação com Brachiaria brizantha em sistema de plantio direto. Engenharia agrícola, 21 (3): 19-33.

Conab

Companhia Nacional do abastecimento. Levantamentos de safras. Disponível em: http://www.conab.gov.br/conabweb/ index.php. Consultado 24/11/2015.

Coelho, A.M.; França, G.E.

1995. Seja o doutor do seu milho - nutrição e adubação. Informativo Agronômico, 17: 1-24.

Demétrio, C.S.; Fornasieri Filho, D.; Cazetta, J.O.; Cazetta, D.A. 2008. Desempenho de híbridos de milho submetidos a diferentes espaçamentos e densidades populacionais. Pesquisa agropecuária brasileira, 43 (12): 1691-1697.

Dourado Neto, D.D.; Palhares, M.; Vieira, P.A.; Manfron, P.A.;

Medeiros, S.L.P.; Romano, M.R.

2003. Efeito da população de plantas e do espaçamento sobre a produtividade de milho. Revista Brasileira de Milho e Sorgo, 2: 63-77.

Empresa Brasileira de Pesquisa Agropecuária - Embrapa.

Centro Nacional de Pesquisa de Solos.

1999. Sistema Brasileiro de Classificação de Solos. Brasília, $412 \mathrm{p}$.
Fancelli, A.L; Dourado Neto, D. 2000. Produção de milho. Guaíba: Agropecuária, 360 p.

Fornasieri Filho, D.

1992. A cultura do milho. Jaboticabal: Funep, p. 273.

Fundaçâo Rio Verde.

2002. Resultados de pesquisa arroz, milho e soja safra 2001/02. Lucas do Rio verde: Fundação Rio Verde, 65 p. (Boletim técnico 5).

Gross, M.R.; Pinho, R.G.; Brito, A.

2006. H. Adubação nitrogenada, densidade de semeadura e espaçamento entre fileiras na cultura do milho em sistema de plantio direto. Ciência e Agrotecnologia, 30: 387-393.

Peixoto, C.M.

2002. Mais plantas, menos espaço. Cultivar, Pelotas, pp. 25-28.

Peixoto, C.M.; Silva, P.R.F.; Rezer, F. \& Carmona, R.C.

1997. Produtividade de híbridos de milho em função da densidade de plantas, em dois níveis de manejo da água e da adubação. Pesquisa Agropecuária Gaúcha, 3: 63-71.

Raij, B. Van; Andrade, J.C.; Cantarella, H.; Quaggio, J.A. (Ed.). 1996. Recomendações de adubação e calagem para o Estado de São Paulo, 2. ed. Campinas: Instituto Agronômico \& Fundação IAC, p. 285, (Boletim Técnico, 100).

Ramalho, M.A.P.

2000. A experimentação em genética e melhoramento de plantas. Lavras, ed. UFLA, pp. 235-245.

Sangoi, L.; Ender, M.; Guidolin, A.F.; Bogo, A.; Kothe, D.M. 2000. Incidência e severidade de doenças de quatro híbridos de milho cultivados com diferentes densidades de plantas. Ciência Rural, 30: 17-21.

Sangoi, L.; Almeida, M.L. de; Lech, V.A.; Gracietti, L.C.; Rampazzo, C.

2001. Desempenho de híbridos de milho com ciclos contrastantes em função da desfolha e da população de plantas. Scientia Agrícola, 58 (2): 271-276.

Silva, P.R.F.; Argenta, G.; Sangoi, L.

2003. Fatores determinantes da escolha de densidade de plantas em milho. In: Reunião Técnica Catarinense de Milho e Feijão, 4., 2003, Lages, SC. Resumos expandidos... Lages: CAV-UDESC, pp. 25-29. 